\title{
A method to determine the ageing rate of thick-film PZT layers
}

\author{
P Glynne-Jones, S P Beeby and N M White \\ Department of Electronics and Computer Science, University of Southampton, Highfield, \\ Southampton SO17 1BJ, UK \\ E-mail:pgj195@zepler.org.uk
}

Received 14 December 2000, accepted for publication 23 March 2001

\begin{abstract}
Screen printed lead zirconate titanate (PZT) layers offer possibilities for both sensing and actuation applications. PZT materials are known to suffer a long-term ageing process that can affect their performance as functional materials. Measuring the rate of this ageing on thick-film piezoelectric materials is difficult due to the presence of a substrate, and suitable methods have not been reported in the literature. A technique for measuring the ageing rate of the $d_{31}$ coefficient is described in this paper, and includes a composite test structure based on a steel substrate. The $d_{31}$ coefficient is found to age at $-4.4 \%$ per time decade after polarization with no external stresses (for PZT-5H, a soft piezoelectric material). A method is also presented for measuring the ageing of the dielectric constant, $K_{33}$, and this is found to have an ageing rate of $-1.34 \%$ per time decade. Techniques for exploring cyclical stress induced ageing of $d_{31}$ are further described, and an interesting linear ageing characteristic is demonstrated.
\end{abstract}

Keywords: $d_{31}$ ageing rate, thick-film piezoelectric constants

\section{Introduction}

Screen printing techniques are an attractive way of producing lead zirconate titanate (PZT) layers, since they enable film thicknesses in excess of $10 \mu \mathrm{m}$ to be routinely deposited. Alternative deposition techniques (sol-gel [1], sputtered [2] or metal-organic chemical vapour deposition (MOCVD) [3]) cannot, at present, deposit material in such thicknesses. Thick films are of particular interest to the field of microelectromechanical systems (MEMS) [4, 5], where their electromechanical properties make them applicable for both sensing and actuating.

It has long been known that piezoelectric ceramics suffer a long-term ageing process after polarization [6]. When these materials are used in sensors and actuators there is a concern that this ageing will decrease the efficiency, sensitivity or accuracy of the devices. It is thus important to characterize the ageing process so that designs can be made that allow for the changes over the lifetime of a device.

Owing to the difficulties in measuring the coupling coefficients of thick-film piezoelectric ceramics, which stem from the presence of an underlying substrate, we have found no previous reports in the literature of any attempts to measure the ageing of these thick-film properties. Making a preliminary study of this effect was thus deemed important. This paper does not attempt to explore the ageing process in detail, but describes a new technique for measuring the ageing of thickfilm PZT, and provides some simple results that will increase confidence in the long-term stability of screen printed PZT thick films.

Methods for studying two types of ageing process are presented here. The first measures the decrease in magnitude of the $d_{31}$ coefficient (which relates stress applied perpendicular to the polarization direction to charge displaced along the polar axis) and the dielectric constant, $K_{33}$, with time after polarization. Finally, a method to examine the effect of cyclical stress on the $d_{31}$ coefficient is explored.

\section{Background}

During the time that a sample of bulk PZT is cooled after polarization, it can be noted that the ageing cycle described in table 1 begins.

Jaffe [7] states that for any of these properties the effect can be expressed for practical time intervals as $K=K_{0}-m \log (t)$ where $K$ is the value of the constant, $K_{0}$ its value at $t=1$, and $t$ the time elapsed since cooling. Jaffe also notes that by mathematical necessity the ageing rate must eventually 
Table 1. Ageing processes (after Jaffe [7]).

Piezoelectric coupling factors Diminish

Dielectric constant

Dielectric loss

Mechanical $Q$

Elastic stiffness

Increase
Frequency constants

diminish (this has been observed after several years of elapsed time), thus the time law is not precisely semi-logarithmic.

Ageing cycles also begin after any subsequent thermal changes, application of strong mechanical stresses, or strong electrical signals; thus when performing experiments these types of disturbance must be avoided, otherwise they will cause smaller ageing cycles that will be superimposed on the cycle of interest [6].

Significant work has been performed to explore the accuracy of this model, and the mechanisms that cause it for both bulk ceramics $[6,8]$ and for thin-film deposited ceramics [9]. The mechanisms proposed generally reflect some form of domain rearrangement [7]. One proposed mechanism [6] is that the loss of activity is due to a decrease in the remnant polarization of the ceramic that occurs to relieve elastic and electrical stresses that are created during the polarization process. A major cause of these stresses is the fact that the unit cell of the PZT material is longer along its polar axis than any of the other allowable directions for the polar axis. Zhang and Zhao [8] describe the ageing of both hard and soft PZT in more detail.

The ageing effect in PZT ceramics is sensitive to the precise composition of the material, for instance the 'hard' high-power material PZT8 has a $d_{33}$ ageing rate of $-6.3 \%$ per time decade, while PZT7A has been tailored for low ageing and is quoted at less than $0.05 \%$ per time decade [10]. Values of a few per cent per decade are typical for bulk materials. Thus to determine experimentally the ageing rate, the measurement method must be sensitive to small changes over relatively long periods of time. The difficulties of measuring the piezoelectric coefficients of thick-film materials are described by GlynneJones et al [11]. Commercially available $d_{33}$ meters tend to be limited to an accuracy of around $\pm 2 \%$ for the types of sample described here, thus the challenge is to find a measurement technique that is both accurate and stable over a period of days.

\section{Development of a thick-film test structure}

Thick-film PZT layers are generally printed on either alumina or, more recently, silicon [12]. The techniques described below use the motion of resonant structures to excite responses in the PZT. To produce structures with a reasonably low (less than $200 \mathrm{~Hz}$ ) resonant frequency and large maximum deflection a test structure based upon a steel substrate was developed.

Figure 1 shows the design of the test structure, which takes the form of a tapered beam clamped at its wide root; table 2 lists its key dimensions. Simple beam theory shows that the tapered shape of the beam means that, for a given deflection caused by a force at the beam point, there will be an equal stress distribution through the thickness of the PZT layer at any point along the beams length (ignoring edge effects).

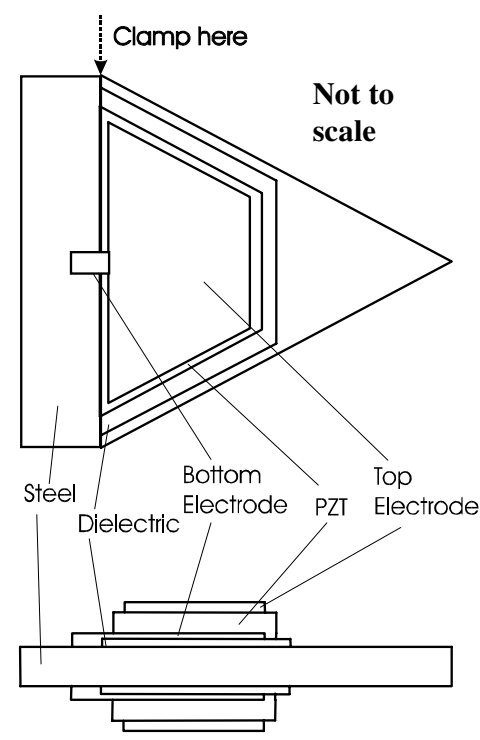

Figure 1. Design of prototype generator.

Table 2. Prototype dimensions.

\begin{tabular}{ll}
\hline Device dimension & Size $(\mathrm{mm})$ \\
\hline Beam length from clamp at root & 23 \\
Beam width at root & 23 \\
PZT width at root & 20 \\
PZT width at tip & 10 \\
PZT length & 10 \\
PZT thickness & 0.07 \\
Electrode width at root & 18.5 \\
Electrode width at tip & 9.5 \\
Electrode length & 9 \\
Bottom electrode thickness & 0.015 \\
Top electrode thickness & 0.015 \\
Distance from beam root to electrode & 0.5 \\
Dielectric width at root & 22 \\
Dielectric width at tip & 11 \\
Dielectric length & 11 \\
Dielectric thickness & 0.020 \\
Steel thickness & 0.100 \\
\hline
\end{tabular}

The steel substrate was formed from $100 \mu \mathrm{m}$ thick, hardened AISI 316 steel. 316 steel has a low carbon content (less than 0.08\%). This is important since, during the PZT firing cycle, the substrate is heated to around $950^{\circ} \mathrm{C}$. Steel with a higher carbon content would tend to oxidize at this temperature. Significant problems were encountered with thermal mismatch between the steel and the thick-film layers. The steel has a coefficient of thermal expansion, $T c=16-18 \mu$ strain $\mathrm{K}^{-1}$, large compared to the typical $6 \mu$-strain $\mathrm{K}^{-1}$ of PZT-5H (the temperature coefficients of both materials, especially the PZT, are actually temperature dependent). During the cooling phase of the film firing (whether electrode, insulator or PZT paste) the ink binds to the substrate, and as cooling continues, the double layer bends in the manner of a bi-morph. To counteract this problem, identical layers were deposited on the front and back sides of the steel, which reduced, but did not eliminate, the problem. 


\subsection{Chemical interaction between PZT layers and steel} substrate

Initially, silver electrodes were printed directly onto the prepared steel, followed by the PZT ink. After firing, the PZT became a discoloured yellow, and the steel in areas adjacent to the PZT had reacted in some manner, causing it to darken. The effect was similar in appearance to that observed by Beeby et al [12]. Beeby's experiments involved printing the same thickfilm ink onto silicon. The effect is thought to be caused by the mobile lead atoms in the PZT reacting with the substrate.

To eliminate the problem of chemical interaction between the steel and the PZT, a dielectric material was chosen to separate the layers. The ink used was IP222L produced by Heraeus Silica and Metals Ltd. This is an $850^{\circ} \mathrm{C}$ firing glaze for Cr-steels. The ink manufacturer recommends printing three separate layers to achieve a printed thickness of $50 \mu \mathrm{m}$ for reliable electrical insulation. It was found that this many layers produced a substrate that was too mechanically stiff to be useful, and experimentation showed that a single layer of around $20 \mu \mathrm{m}$ was sufficient to prevent damaging interaction between the steel and the PZT.

\subsection{Electrodes}

The bottom electrode was formed from a commercial cermet ink, which can withstand the subsequent firing of the PZT layers. To prevent significant interaction between the PZT and the electrode, a low-migration silver/palladium ink, 9633B, produced by Electro-Science Laboratories Inc. was used.

Initially top electrodes were also fabricated with a cermet ink; however, these are mechanically stiff and substantially reduce the proportion of elastic strain stored in the PZT when the beam is flexed. A silver loaded polymer ink (ESL 1107) was therefore chosen for this purpose.

\subsection{Piezoelectric layer}

Commercial piezoelectric inks are not currently available. The films were based on a PZT-5H powder of particle size $6 \mu \mathrm{m}$ supplied by Morgan Electroceramics Ltd. The composition of the PZT film used here was identical to that described by Beeby et al [12].

Standard thick-film processing techniques normally produce layers around 8-15 $\mu \mathrm{m}$ thick. In this case, a larger thickness was required to produce a higher electrical signal-tonoise ratio, and avoid any pin-hole defects that would prevent polarization at high field strengths. To produce a $70 \mu \mathrm{m}$ thick fired layer, a wet thickness of around $140 \mu \mathrm{m}$ was required, assuming a shrinkage of $50 \%$ during the drying and firing cycles. Using a thick emulsion layer on the screen, and a thick mesh would produce such a layer, but the surface of the resultant print would not be even due to the coarse mesh.

To produce a reliable thick layer it was necessary to print several layers on top of each other, firing once every 50-70 $\mu \mathrm{m}$ to prevent cracking that can occur through shrinkage of the unfired film. The exact sequence of printing, drying and firing used is described below.

\subsection{Polarization}

To polarize the piezoelectric layer, samples were heated to $150{ }^{\circ} \mathrm{C}$ then left for 10 minutes to become thermally stable. An electric field of $3.5 \mathrm{MV} \mathrm{m}^{-1}$ was then applied across the electrodes. The field was maintained for 65 minutes in total, 35 minutes at the high temperature, followed by another 30 minutes as the sample was allowed to cool down. Since heating one side of the substrate results in heating of the other side, it was necessary to polarize both sides at once. The long polarization time is shown by Dargie [13] to give increased activity.

\subsection{Processing steps}

The overall procedure for producing the prototype devices is outlined below.

Sheets of steel (as described above) were cut into $50 \mathrm{~mm}$ squares using a guillotine. Both sides were roughened with P1000 emery paper, then degreased in acetone.

The dielectric ink was printed on each side of the substrate using a DEK-1750 printer. Each side was dried at $200^{\circ} \mathrm{C}$ for 15 minutes after printing; the reverse face was printed after the front face had been dried. Both sides were then fired simultaneously in air using a BTU Belt furnace (model QA416-54) using a temperature profile with a peak temperature of $890{ }^{\circ} \mathrm{C}$ for 10 minutes, total firing time 60 minutes. The silver/palladium electrode was printed and fired on each side of the substrate using the same steps as for the dielectric ink.

The PZT paste was deposited on each side in the following sequence: double print, dry, double print, dry, fire, double print, dry, double print, dry, fire. (Double printing involves printing, then printing again over the wet layer, to ensure full coverage. This is necessary when the ink fails to print evenly on the first pass - a problem with the current formulation of PZT ink).

The silver top electrode was printed, and cured at $200^{\circ} \mathrm{C}$ for 60 minutes after printing. A guillotine was used to separate each device from its neighbours on the substrate. Wires ( $0.2 \mathrm{~mm}$ diameter insulated copper) were then soldered to the electrodes, and the device was polarized as described above.

\section{Experimental apparatus}

The results described in this section have been obtained using the apparatus shown in figure 2. A Goodman V.50 mark 1 (model 390) vibration generator (shaker) was used to supply mechanical vibrations to the samples under test. The shaker was driven by a Ling PA50VA valve amplifier. The amplifier was driven from the frequency source incorporated into a Hewlett Packard HP35660A dynamic signal analyser.

A vernier screw gauge was mounted on a plate that is attached by threaded steel rods to the shaker. The screw gauge has a resolution of $0.01 \mathrm{~mm}$, and allowed the tip amplitude of a vibrating beam to be measured. Figure 3 shows the shaker, suspension, clamp block, accelerometer (Bruel \& Kjaer, type 4369), screw gauge and a typical sample as they are mounted together.

To measure the charge displaced by the PZT and the charge signal from the accelerometer, Kistler charge amplifiers 


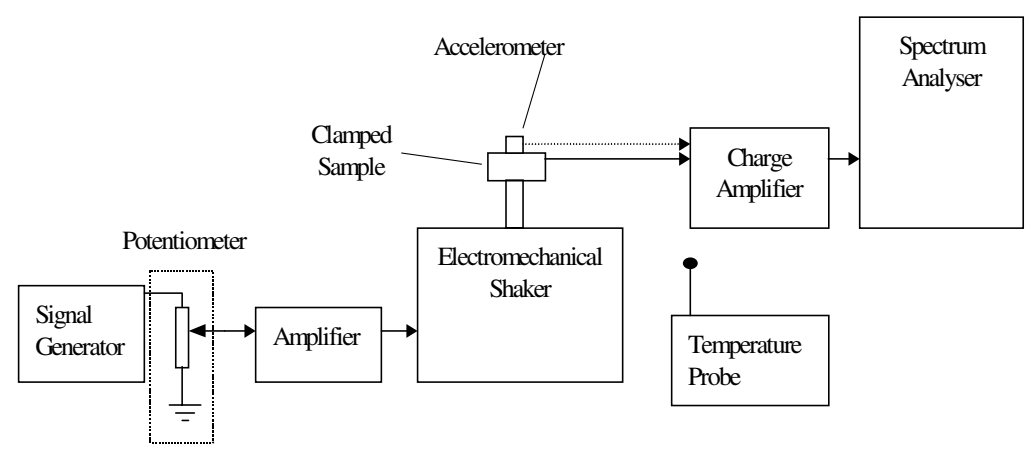

Figure 2. Experimental set-up.

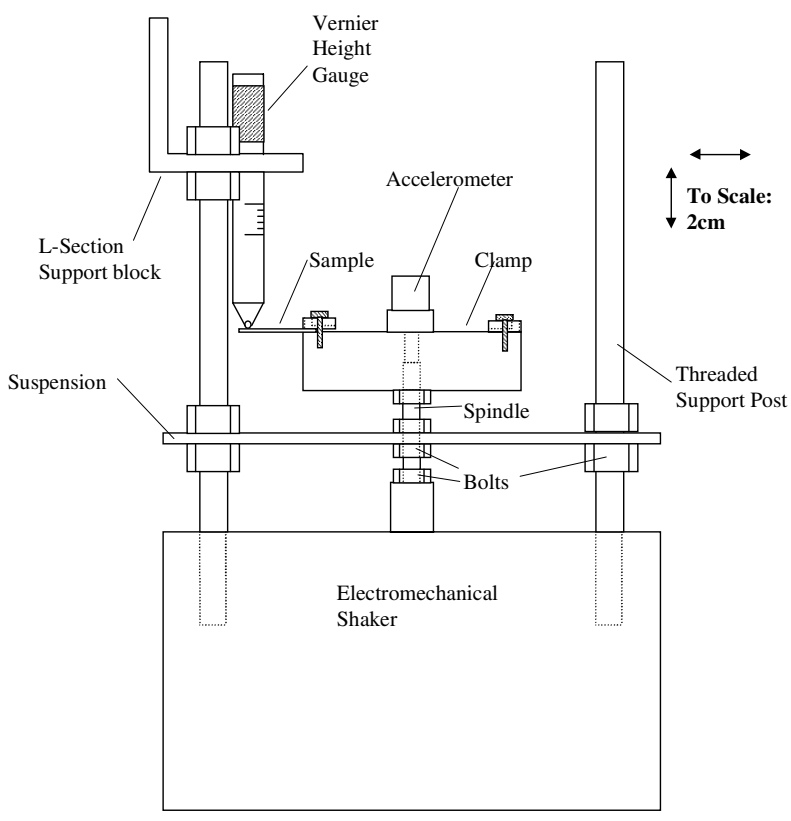

Figure 3. Shaker, clamp and vernier gauge arrangement.

(model 5001) were used. The outputs from the charge amplifiers were both monitored by the signal analyser.

In some experiments, a mass was attached to the tip of a sample by using a short thread to hold a pair of nuts on either side of the sample.

\section{Compensation of charge amplifier response}

During experimentation, it was found necessary to measure two quantities of charge simultaneously. To do this two charge amplifiers were used. These, however, were not sufficiently stable with either temperature or over the long duration of the experiment. To eliminate any long-term drift the following procedure was devised.

Assume that the two charge amplifiers have no zero offset error, but have unknown amplification factors $K$ and $L$ respectively (unknown due to poor calibration or long-term drift). We wish to determine the ratio, $R$, between the charge signals being produced by two samples.

The charge amplifiers are connected to the samples and a reading is taken. If the two samples yield charges $a$ and $b$ respectively, then the outputs from the charge amplifiers are given by $\alpha=K a$ and $\beta=L b$. The wires are now reversed, and another reading is taken. Since the reading is taken after the first one, the charge signals will have changed, but their ratio, $R$, will remain constant. If we denote these second signals as $c$ and $d$ respectively, then the outputs from the charge amplifiers are now given by $\chi=K c$ and $\delta=L d$.

It can be shown that the ratio between the input signals, $R$, can be determined by the following equation that is in terms of the measured outputs from the charge amplifiers, and is independent of $K$ and $L$.

$$
R=\sqrt{\frac{\alpha \delta}{\beta \chi}} .
$$

\section{Measuring the $d_{31}$ coefficient of the test structures}

The methods described below for determining the ageing rates of the PZT layers in the test structures do not produce an absolute measure of the $d_{31}$ coefficient. This was measured as follows.

The $d_{31}$ coefficient was measured using the direct effect (see Glynne-Jones et al [11] for a discussion of methods for measuring the piezoelectric coefficients of thick-film samples). A test structure was polarized and fixed in the clamp as described above. The beam was shaken, and the drive to the shaker adjusted until the beam amplitude (measured using the vernier screw gauge) reached a predetermined level. A charge amplifier was used in conjunction with the signal analyser to determine the charge displaced at the point of maximum displacement for the beam. Figure 4 shows a graph of charge displaced against excitation amplitude for a sample at a temperature of $22^{\circ} \mathrm{C}$. A least squares method was used to estimate the slope of the graph. A finite element analysis was performed using the model shown in figure 5 with the ANSYS package to calculate the average longitudinal stress in the layer. These data were combined with the surface area of the electroded PZT layer, to calculate the $d_{31}$ coefficient

$$
d_{31}=\frac{\text { charge }}{\text { area } \times \text { stress }} .
$$

The $d_{31}$ values for a set of four samples were measured and found to yield an average value of $15 \mathrm{pC} \mathrm{N}^{-1}$. 


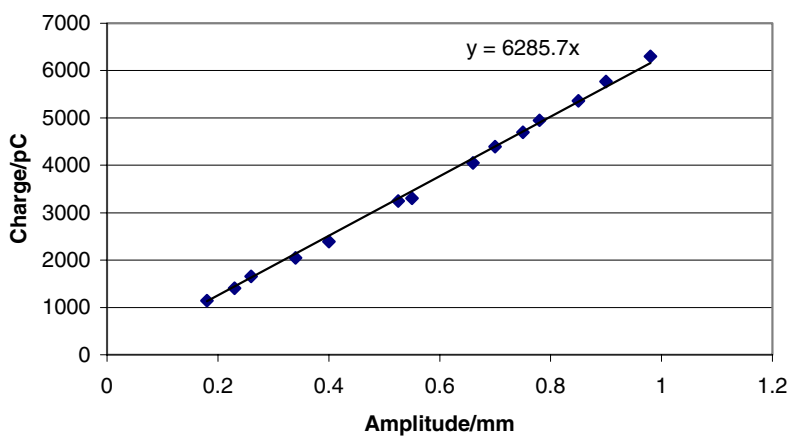

Figure 4. Graph of charge displaced against amplitude, to find $d_{31}$.

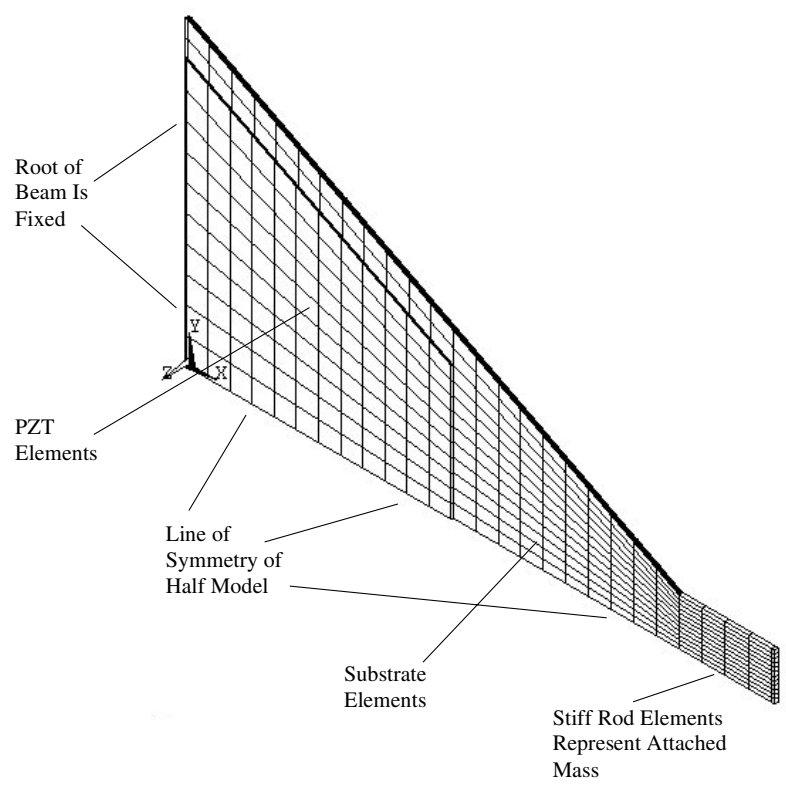

Figure 5. Finite element mesh model of tapered test structure.

\section{Temporal ageing after polarization}

When the test structures described above are mounted in the clamp and shaken the resulting signal at the electrodes is a function of the $d_{31}$ coefficient of the PZT. Thus by examining how this signal changes with time after polarization, the ageing of the $d_{31}$ coefficient can be observed. Similarly by measuring how the capacitance of the sample varies after polarization, the ageing rate of the dielectric constant can be deduced.

\subsection{Experimental procedures}

Masses were attached to the tips of two beams as described above. Preliminary experiments showed that both the base excitation supplied by the shaker and the response of a clamped sample to shaking varied with environmental temperature; this variation obscured the ageing present in the sample (see figure 7). To compensate for this effect, a reference sample was used. The reference sample was mounted at the opposite end of the clamp to the main sample. This reference sample was prepared in an identical manner to the main sample, except that it was polarized one month before the experiment. If the system is assumed to be linear, then if the base acceleration is increased, the ratio between the signals from the reference sample and the main sample should remain unchanged. Similarly, the reference sample should compensate for any linear temperature dependence of the $d_{31}$ coefficient with temperature. By polarizing the reference sample one month before the experiment, it should not age more than a further $-0.12 \%$ over the course of a 2 day experiment (given the rate of ageing found experimentally).

The shaker was driven by the amplifier and frequency source described above. The frequency of excitation was set at $60 \mathrm{~Hz}$, around two-thirds of the $90 \mathrm{~Hz}$ natural frequencies of the sample and reference beams. Preliminary experiments had been performed operating at the resonant frequency of the sample; it was found, however, that as temperature variations occurred the natural frequencies of the beams would change, causing large variations in the beams' amplitudes. It was also found that at resonance the positions of the connecting wires had large effects on the beams' amplitudes, leading to unreliable results (at resonance the amplitude is controlled by the amount of damping present; the connecting wires cause a significant part of this damping). Operating at $60 \mathrm{~Hz}$ is a compromise between using higher frequencies with the associated damping problems, and lower frequencies which would produce a smaller signal to noise ratio.

The main sample was placed in the clamp immediately after it was polarized. The amplitude of the acceleration applied by the shaker was set to $28 \mathrm{~m} \mathrm{~s}^{-2}$ (measured using the accelerometer), which produced a strong signal without being large enough to cause mechanical change in the test apparatus. The amplitude of the beam at this excitation was measured as $0.3 \mathrm{~mm}$. Using the finite element model described above, it was found that this results in a maximum longitudinal stress in the PZT layer of 2.7 MPa. This is in the linear range described for bulk PZT5A [10], which is given a maximum rated static compressive stress perpendicular to the polar axis of $13.8 \mathrm{MPa}$. It is thus reasonable to assume that the device is operating in its linear range, and that the shaking should not contribute any stress-induced ageing cycles.

To measure the charge displaced by the two beams a pair of Kistler charge amplifiers (model 5001) were used. The charge amplifiers were set to a sensitivity of $1000 \mathrm{pC} \mathrm{V}^{-1}$ with a feedback resistance of $10^{9} \Omega$. The signal analyser monitored the output from the charge amplifiers, and was used with a flat-top (or sinusoidal) windowing function, which gave better amplitude accuracy than the other available functions. The signal analyser was set to a sampling rate of $256 \mathrm{~Hz}$, with a total number of 512 samples. The analyser performed fast Fourier transforms to show the average signal amplitude over the sampling period.

Once the sample was polarized and clamped, and the shaker activated, then a set of measurements was taken periodically. To take a set of measurements, first the charge signals were measured with the main sample connected to charge amplifier ' $A$ ', and the reference connected to charge amplifier 'B'. A reading was taken simultaneously of both quantities. (The signal analyser has two input channels that are logged concurrently. Both can be paused simultaneously to allow this measurement). The connecting leads were then switched so that the main sample was connected to charge amplifier ' $\mathrm{B}$ ', and the reference connected to charge amplifier ' $\mathrm{A}$ '; readings of the charge signals were again taken 


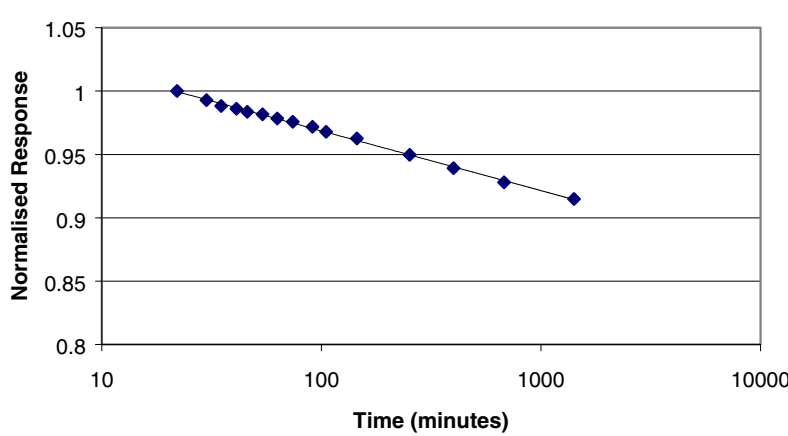

Figure 6. Graph of normalized $d_{31}$ versus time after polarization.

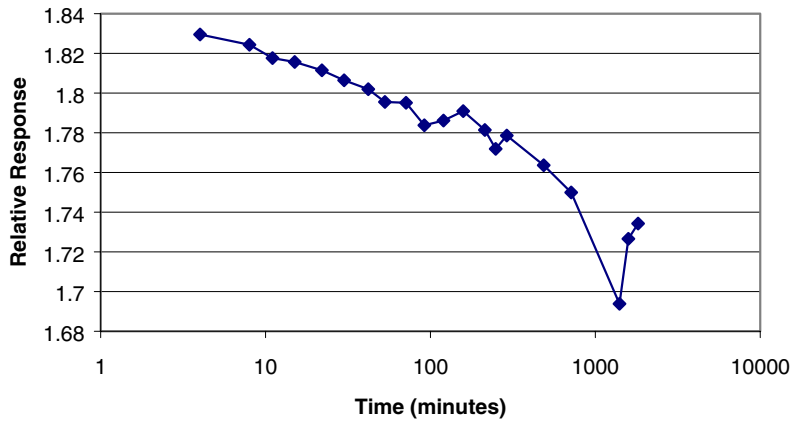

Figure 7. Graph of $d_{31}$ response versus time without compensation.

simultaneously. Equation (1) was then used to determine the ratio of sample response to reference response. The experiments described here continued for up to 2 days, with the interval between readings increasing to provide suitable data to plot a semi-logarithmic graph of the results.

The ageing of the dielectric constant of the PZT layer was examined as follows: a sample was polarized as described above, then the capacitance of the sample was measured periodically using a Wayne-Kerr automatic $L C R$ meter (model 4250). To compensate for any linear dependence on environmental conditions, the response of the sample was compared to a reference sample that had been pre-aged for one month before the experiment, as described above. Whenever the sample capacitance was measured, the reference sample was tested shortly afterwards, so that the two measurements related to nearly the same instant in time.

\subsection{Results and discussion}

Figure 6 shows how the ratio of sample response to reference response (for the $d_{31}$ experiment) varied with time after polarization. The data are normalized (linearly scaled) so that the initial value of the ratio has a value of one. The figure includes a linear best-fit line, found using a least squares method. The graph exhibits a linear semi-logarithmic relationship that corresponds well to the type of ageing found with bulk samples. Figure 7 shows a typical response of a sample without compensation by a reference beam; a reference beam is clearly useful in compensating for environmental and excitational variations.

Three other samples were also tested; the results are listed for each side of each sample in table 3 . The average ageing rate of the layers was $-4.40 \%$ per time decade, with a standard
Table 3. $d_{31}$ ageing rates of samples.

\begin{tabular}{lll}
\hline & \multicolumn{2}{c}{$\begin{array}{c}d_{31} \text { ageing rate } \\
(\% \text { decay/decade })\end{array}$} \\
\cline { 2 - 3 } & Side one & Side two \\
\hline Sample 1 & 4.72 & 4.93 \\
Sample 2 & 4.69 & 4.59 \\
Sample 3 & 4.26 & 4.35 \\
Sample 4 & 3.94 & 3.73 \\
\hline
\end{tabular}

Table 4. $K_{33}$ ageing rates of samples.

\begin{tabular}{ll}
\hline Sample & $\begin{array}{l}\text { Dielectric constant, } K_{33}, \\
\text { ageing rate (\% decay/time decade) }\end{array}$ \\
\hline 1 & $1.11 \%$ \\
2 & $1.33 \%$ \\
3 & $1.37 \%$ \\
4 & $1.54 \%$ \\
Mean value & $1.34 \%$ \\
\hline
\end{tabular}

deviation of 0.41 . The variation observed between samples is not unexpected; it is stated by the IRE standard for the measurement of piezoelectric crystals [14] that parameters can vary by up to $20 \%$, even in ceramics of known composition and high density. The thick-film printing process is also very sensitive to processing conditions, and individual samples are subject to fluctuations even within a given batch. The rate of ageing is similar to that described by Morgan Electroceramics [10] for the $d_{33}$ coefficient of PZT-5H, reported at $-3.9 \%$ per decade.

The results of this experiment indicate that thick-film PZT materials can be used in future designs with the confidence that they will not age significantly faster than traditional bulk materials (under conditions of low stress, and low electrode voltage).

Figure 8 shows how the ratio of sample capacitance to reference capacitance varied with time after polarization. The data are normalized (linearly scaled) so that the initial value of the ratio has a value of one. The figure includes a linear best-fit line, found using a least-squares method. The figure again exhibits a clear linear semi-logarithmic relationship that corresponds well to the type of ageing found with bulk samples. The results for four samples are presented in table 4 . The mean ageing rate is found to be $-1.34 \%$ per time decade. This is higher than the value of $-0.6 \%$ reported by Morgan Electroceramics for bulk PZT-5H [10].

\section{Ageing caused by cyclic stress}

The experiments described in this section attempt to examine the rate of ageing induced by cyclical stress on thick-film samples. The response of a sample that is caused to oscillate at large amplitudes is examined over a large number of cycles. The response is compared to a reference sample with less mass, that oscillates at a smaller amplitude, and hence less stress. The method is found to give unreliable results at the high amplitudes required to cause classical semi-logarithmic ageing of the sample; lower amplitudes are, however, found to cause a small linear ageing effect that is presented as interesting and potentially important. 


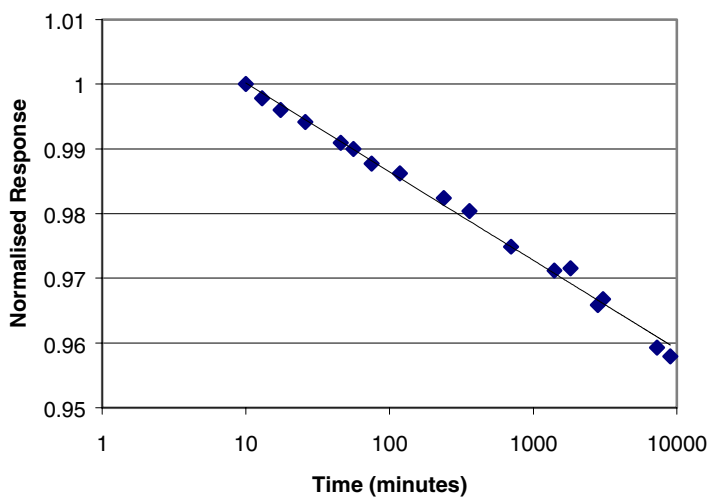

Figure 8. Graph of normalized $k_{33}$ versus time after polarization.

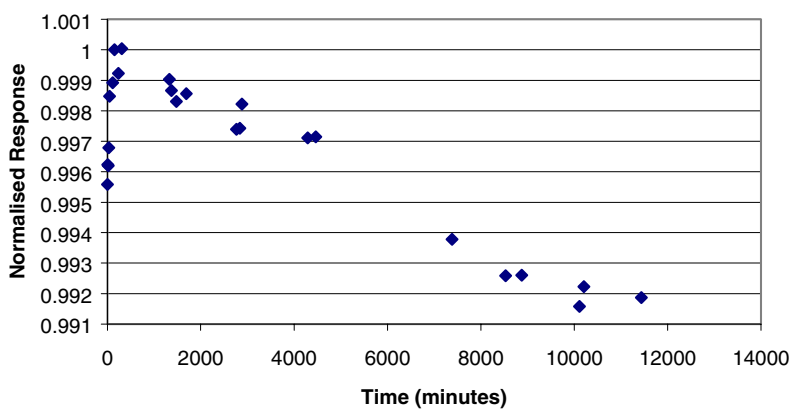

Figure 9. Ageing of response of a sample with amplitude $0.51 \mathrm{~mm}$.

\subsection{Method}

Sample and reference composite beams were prepared and equipped with wires as described in the previous experiment. Both beams were polarized, then left for 60 days so that the amount of natural ageing over the course of the experiment would be negligible (over the course of an 8 day experiment the $d_{31}$ of these samples will decrease by only a further $0.2 \%$, assuming an ageing rate of $-4 \%$ per decade).

The two beams were mounted in the shaker rig as before. In this experiment, a tip mass was placed on the sample beam, but not the reference beam. This means that when the clamp is shaken, the sample oscillates with a much higher amplitude than the reference beam. The sample beam was thus being shaken at an amplitude that may cause it to age due to the cyclic stresses induced in it. The reference beam should not age in this manner, and should allow for compensation of any variation in the excitation amplitude and linear temperaturerelated coefficient changes.

For the reasons described in the previous experiment, the shaker was operated at $55 \mathrm{~Hz}$. Samples were tested at two different excitation amplitudes. Table 5 lists the beam amplitudes, and the associated base excitation, and the maximum stress in the PZT at these amplitudes predicted using the FEA model. The longitudinal stresses predicted in the PZT layer would place the samples in the linear region of operation were they formed from bulk material (PZT-5A has a maximum rated compressive stress perpendicular to the polar axis of $13.8 \mathrm{MPa}$ ).

Larger beam amplitudes were investigated, but it was found that at such amplitudes there were large variations in the
Table 5. Beam amplitudes for ageing experiment.

\begin{tabular}{lll}
\hline $\begin{array}{l}\text { Beam } \\
\text { amplitude } \\
(\mathrm{mm})\end{array}$ & $\begin{array}{l}\text { Magnitude } \\
\text { of base } \\
\text { acceleration } \\
\left(\mathrm{m} \mathrm{s}^{-2}\right)\end{array}$ & $\begin{array}{l}\text { Maximum longitudinal } \\
\text { stress in } \\
\text { the PZT layer } \\
(\mathrm{MPa})\end{array}$ \\
\hline 0.51 & 48 & 4.8 \\
0.85 & 79 & 8.1 \\
\hline
\end{tabular}

Table 6. Stress induced ageing of samples.

\begin{tabular}{lll}
\hline Sample & $\begin{array}{l}\text { Ageing rate } \\
\left(\% \text { min }^{-1}\right)\end{array}$ & $\begin{array}{l}\text { Ageing rate } \\
(\% / m i l l i o n \\
\text { cycles })\end{array}$ \\
\hline Amplitude $=0.85 \mathrm{~mm}$ & $-7.45 \times 10^{-5}$ & -0.023 \\
Amplitude $=0.51 \mathrm{~mm}$, side 1 & $-7.51 \times 10^{-5}$ & -0.023 \\
Amplitude $=0.51 \mathrm{~mm}$, side 2 & $-7.45 \times 10^{-5}$ & -0.023 \\
\hline
\end{tabular}

results that obscured any meaningful trends. These variations could be due to the large amount of base excitation required to achieve these beam amplitudes in a sub-resonant beam causing mechanical changes in the clamping arrangement, or perhaps the strength of the thick-film layer in tension or compression is exceeded.

The charge signals from the two samples were fed into the pair of charge amplifiers. These were set to sensitivities of $1000 \mathrm{pC} \mathrm{V}^{-1}$ and $50 \mathrm{pC} \mathrm{V}^{-1}$ for the sample and reference beams respectively. The output from the charge amplifiers was monitored by the signal analyser described above. As before, a flat-top (or sinusoidal) windowing function was used. The signal analyser was set to a sampling rate of $128 \mathrm{~Hz}$, with a total number of 512 samples.

The shaker was activated, then at intervals a set of measurements were taken. Sets of simultaneous measurements were taken as described above for the temporal ageing experiment, and the ratio of sample response to reference response was again determined using equation (1). Experiments were conducted for up to 9 days in duration.

\subsection{Results and discussion}

Figure 9 shows how the ratio of sample to reference response varied with shaking time for a beam shaken at an amplitude of $0.51 \mathrm{~mm}$. In this graph, there is an initial period (around 3 hours) of increase in the normalized response of the sample, followed by a steady linear decrease. The reason for this increase is unclear; a possible reason is stress-induced fatigue of the steel substrate.

The rates of ageing (after the initial increase period) of the samples are listed in table 6. It is interesting to note that the rate of ageing is the same for both of the beam amplitudes examined here. The total amount of ageing observed over the course of this experiments is less than one per cent. This is much smaller than the $10 \%$ total ageing (in a similar time span) observed over the course of the after polarization experiments described above. Note that previous work [11] to measure the piezoelectric coefficients of thick-film PZT has achieved accuracies of only a few per cent for each reading, and that the experiments describe here offer an order of magnitude increase in experimental accuracy. 
The ageing in the samples' responses has several possible sources: a decrease in the activity of the PZT layer, damage to the PZT layer, or a stress-induced stiffening of the steel substrate. The stresses in the PZT are at a low level compared to the level where the onset of non-linear response is seen in bulk PZT. The region of non-linear response is closely associated with the range of stresses that will induce ageing cycles in a sample [6]. That the ageing is linear with respect to time also indicates that the observed results are not a result of classical domain rearrangement ageing of the PZT [6]. We thus tentatively deduce that the ageing is due to work hardening of the steel or cyclical fatigue of the PZT.

Since the experimental set-up has proved unsuitable for generating larger stresses within the PZT layer, further insight into classical stress-induced ageing of the screen-printed PZT will require a different approach.

\section{Conclusions and further work}

A technique for measuring the ageing rate of the $d_{31}$ coefficient of a PZT thick-film sample has been presented. The method is found to be reliable, and sufficiently accurate for observing the decaying response. The accuracy obtained is of an order of magnitude higher than that reported previously. The $d_{31}$ coefficient is found to age at $-4.4 \%$ per time decade (for PZT$5 \mathrm{H})$. A method is presented for measuring the ageing of the dielectric constant, $K_{33}$, and found to show an ageing rate of $-1.34 \%$ per time decade. Future studies into the effect of polarization conditions and other processing parameters on the rate of ageing are anticipated.

A technique for exploring the ageing induced by cyclical stress has also been described. The method has been found to be unreliable at the higher stresses required to induce classical ageing; however, at lower amplitudes, a small linear ageing effect has been found that warrants further study.

\section{Acknowledgments}

The authors wish to thank the Engineering and Physical Sciences Research Council (EPSRC) for their financial support under grant number GR/M35086. We also gratefully acknowledge the support and assistance given to us by Morgan Electroceramics Ltd.

\section{References}

[1] Aoki K, Fukuda Y and Nishimura A 1993 Japan. J. Appl. Phys. 32 4147-9

[2] Tvarozek V, Novotny I, Cerv-en I, Kovac J and Lacko T 1992 Sensors Actuators A 30 123-7

[3] Hendricks W C, Desu S B, Si J and Peng C H 1993 Mater. Res. Soc. Symp. Proc. vol 310 (Pittsburgh, PA: Materials Research Society) pp 241-7

[4] Muralt P 1997 Integrated Ferroelectrics 17 297-307

[5] Deschanvres J L, Rey P, Delabouglise G, Labeau M, Joubert J C and Peuzin J C 1992 Sensors Actuators A 33 43-5

[6] Mason W 1955 J. Acoust. Soc. Am. 27 73-84

[7] Jaffe B, Cook W and Jaffe H 1971 Piezoelectric Ceramics (New York: Academic)

[8] Zhang Q M and Zhao J 1999 IEEE Trans. Ultrason. Ferroelectr. Freq. Control 46 1518-26

[9] Shepard J F, Chu Fan and Trolier-McKinstry S 1999 J. Appl. Phys. 85 6711-6

[10] Piezoelectric Ceramics Data Book for Designers 1999 (Morgan)

[11] Glynne-Jones P, Beeby S P, Dargie P, Papakostas T and White N M 2000 Meas. Sci. Technol. 11 526-31

[12] Beeby S P, Blackburn A and White N M 1999 J. Micromech. Microeng. $9218-29$

[13] Dargie P G 1998 A study of the effect of process parameter variation upon the characterization of screen printed piezoelectric materials MPhil Thesis University of Southampton

[14] IRE Standards on Piezoelectric Crystals: Measurements of Piezoelectric Ceramics 1961 (The Institute of Electrical and Electronic Engineers) 